\title{
Face Recognition System based on Single Image under Varying Illuminations and Facial Expressions
}

\author{
Amal M. S. Algharib \\ Computer Engineering \\ Ankara Yildirim Beyazit University \\ Ankara / Turkey
}

\author{
Shafqat Ur Rehman \\ Computer Engineering \\ Ankara Yildirim Beyazit University \\ Ankara/ Turkey
}

\begin{abstract}
Small Sample Size (SSS), varying illuminations and facial expressions are three important challenges for any face recognition system. Many studies have addressed these challenges by using some different pre-processing and feature extraction techniques, but a lot of these techniques are still facing the performance issues with these challenges. Therefore a reliable system based on single image is presented in this paper to deal with the above challenges together by using robust pre-processing chain with a powerful feature extraction method, where the proposed pre-processing chain consists of Difference of Gaussian (DoG) filter and waveletbased normalization with histogram equalization technique, this chain has been selected carefully based on the compatibility between their elements and their effectiveness in dealing with illumination effects, local shadows and highlights, then the Regularized Linear Discriminate Analysis (RLDA) is used as a robust feature extraction, the RLDA very effective method in the case of small sample size and facial expressions due to the addition of the regularized parameter to the traditional method (LDA), the extracted features have classified by using different distance classifiers (cosine and correlation classifiers).
\end{abstract}

The proposed system was tested by using three standard databases (Extended Yale-B, JAFFE, AR) which contain the above challenges.

\section{Keywords}

Face recognition, Varying illuminations, small sample size, Facial expressions, Regularized Linear Discriminate Analysis.

\section{INTRODUCTION}

Recently, face recognition attract attention more than other identification methods such as (fingerprint, iris, etc.), especially during the last decades. Because the human face is unique, contains many features and accurate details which give high and reliable identification rates more than the other identification methods. In addition, identification systems based on fingerprint, iris and gene structure (DNA fingerprint) depend on human cooperation, although there are reliable methods to recognize persons which perform well under certain conditions. But these methods still facing many problems such as small sample size, varying illuminations, facial expressions, occlusion, etc. according to study [1], the performance of face recognition systems affected by varying illuminations especially when the number of training samples is small. However, many methods have been suggested to solve the illumination problems such as such as (HE, LT, GIC, DGD, LoG, SSR, GHP, SQI, LDCT, LTV, LN,TT) [2,3], 2DDCT with brightness correction[4], these methods still need to improve to get the desired performance. On the other hand, identify facial expressions addresses the problem of classification facial expressions into categories such as (angry, natural, sad, etc.).The facial expression recognition considered as one of the most important factors, where many of applications depend on a facial expressions classification such as machine learning, robotics and virtual reality and intelligent human-computer interaction (HCI), etc.

Over the past two decades, there are a lot of methods and studies have been done on facial expressions $[5,6]$. But these methods still have limited performance, whether in the case of facial expressions recognition or face recognition under different facial expressions.

In this work, we deal with the above mentioned challenges together by using robust and fast pre-processing chain in order to improve the quality of the originally given images by eliminating the effects of the varying illuminations, the process of extracting features has performed by Regularized Linear Discriminate Analysis (RLDA). Then the extracted features have classified by using different distance classifiers (cosine and correlation classifiers).

In order to prove the efficiency of proposed system, some experiments have performed and were compared with some previous studies that are using illumination normalization techniques. The "Extended Yale-B" database provides a perfect test case which contains varying Illumination conditions [7], it has utilized in our study. Also, for facial expression challenges, we used the JAFFE database [8] in our experiment; lastly the AR database has used to test our system under different illumination conditions and facial expressions [9].

\section{PREPROCESSING CHAIN}

In this part, we describe a simple and effective pre-processing chain which consists DoG filter, wavelet-based normalization with the histogram equalization technique, this chain is effective to deal with illumination effects, local shadows and highlights.

In the next section we will explain each component of this chain.

\subsection{Difference of Gaussian (DoG) Filter}

The first step of our preprocessing chain is applying DoG filtering; the DoG Filter is a grayscale image enhancement method that includes subtracting one blurred image from another less blurred image by performing two different Gaussian blurs [10]. DoG filtering will be first applied to reduce the local variations in face imagery and enhance the edges, where the details of edge are informative and enhance those details for all sub-bands would be helpful.

The (DoG) filter[2] consist of tow inner filters (low-frequency filter and high-frequency filter).These filters remove the high and low frequency details that often include random noise and 
enhance the image quality. The DOG filter on the image $I(x, y)$ is defined as Equation (1):

$$
I^{\prime}(x, y)=I^{*} \frac{1}{2 \pi \sigma_{1}^{2}} e^{-\frac{x^{2}+y^{2}}{2 \sigma_{1}^{2}}}-I^{2} \frac{1}{2 \pi \sigma_{2}^{2}} e^{-\frac{x^{2}+y^{2}}{2 \sigma_{2}^{2}}}
$$

Where $\sigma 1$ inner filter ( $\sigma_{1} \leq 1$ pixel) and $\sigma 2$ inner filter ( $\sigma 2$ from 2-4 pixels or more).

Based on research paper [2].This filter has a great and important effect to remove illumination changes. We find that $\sigma_{1}=1$ and $\sigma_{1}=2$ give best results.

\subsection{Wavelet-based Normalization \\ Technique}

The second step after applying the DoG filter on the image is using the wavelet-based normalization technique, where this technique suggests split an image(decompose) by using DWT (2D discrete wavelet transform) to four sections (sub-bands): low-low(LL), low-high(LH), high-low(HL) and high-high(HH) sub-bands [11], see figure 1, where the LL is the lowfrequency component and can be split into higher levels of decomposition while the three other sub-bands are the highfrequency components.
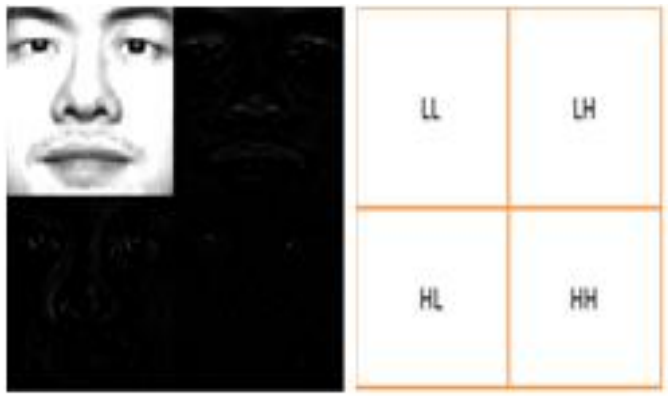

Fig 1: One-level 2-D discrete wavelet transforms

The sub-band analysis of images requires extraction forms from three sub-bands (LH,HL,HH) that contain horizontal, diagonal, vertical details, these sub-bands are less affected by illumination variations, unlike LL sub-bands which more effects by illumination variations.

The next step is execute the photometric normalization procedure by using histogram equalization technique to enhance the image's contrast depending on the density distribution, this method suggests converting the distribution of pixel intensity values of the image $\mathrm{I}(\mathrm{x}, \mathrm{y})$ to uniform distribution, the purpose of histogram equalization is to redistribute the original histogram using the entire range of discrete levels of the image and improve the global image's contrast, the histogram equalization technique has applied to LL sub-band because of the LL sub-band is more sensitive to the illumination than the three other sub-bands. Histogram equalization can be defined as follows:

$$
\mathrm{P}(\mathrm{i})=\mathrm{ni} / \mathrm{TN}
$$

Where: $\mathrm{I} \in 0, \mathrm{i}, \ldots, \mathrm{k}-1$ gray level and $\mathrm{TN}$ is a total number of pixels in the image. Transformation to a new intensity value is defined by:

$$
I^{\prime}=\sum_{i=0}^{k-1} \frac{n_{i}}{N}=\sum_{i=0}^{k-1} p(i)
$$

The output values will be in domain of $[0,1]$. To obtain pixel values into the original domain, it must be rescaled by the $\mathrm{k}-1$ value. Figure 2 shows effects of the histogram equalization technique on the images.
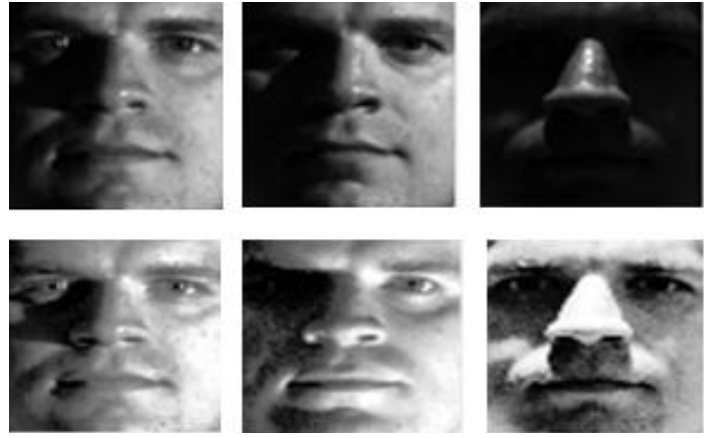

Fig 2: Impact of HE: original image (upper row), histogram equalized on image (lower row).

\subsection{Steps of our Pre-processing Chain}

1- Apply DoG filter on an input image.

2- Apply wavelet based normalization technique and decomposed the input image to four sub-bands (LL, LH,HL,HH).

3- Normalize the LL sub-bands by using histogram equalization technique.

4- Take the inverse of DWT.

The visual steps of the proposed preprocessing chain are illustrated in figure 3 and figure 4 illustrates the effects of proposal preprocessing chain on the images.

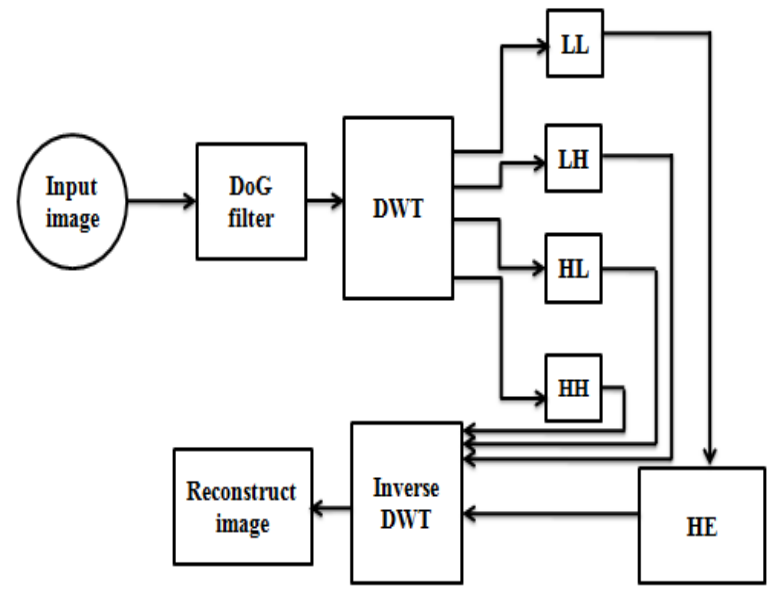

Fig 3: The visual steps of preprocessing chain
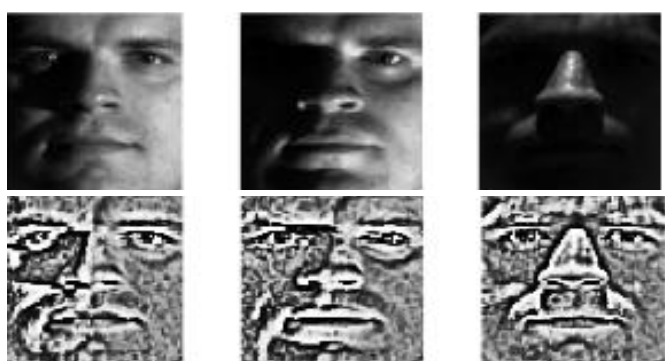

Fig4: Effects of proposal preprocessing chain on the images (upper row -original images, lower row -

Processed images) 


\section{SYSTEM FRAMEWORK}

The proposed system consists of two basic phases (training phase, testing phase).In the training phase, the pre-processing chain was applied to the training images and converted them to vectors, then we have used RLDA technique as feature extraction to extract the important features and create the features matrix [12], the features matrix has projected to vectors matrix, Lastly, both features matrix and projected training vectors matrix are stored in order to use them later in the testing phase.

In the testing phase, the same pre-processing chain was applied to the testing images and converted them into vectors matrix, then we projected the testing vectors matrix with features matrix which obtained on from the training phase. Lastly, the distance between projected training vectors and projected testing vectors has calculated by using different distance classifiers (cosine and correlation classifiers) as shown in figure 5 .

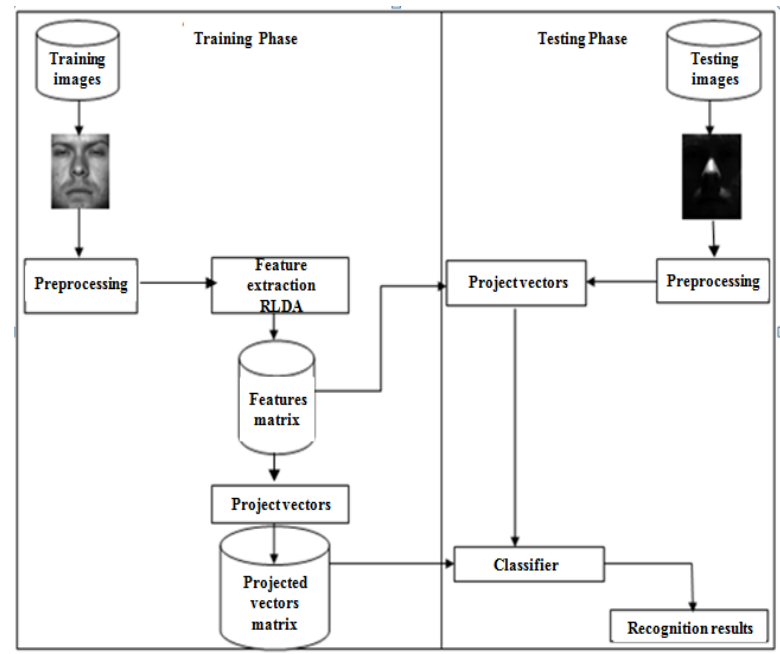

Fig5 .Overview of the proposed system

\section{EXPERIMENTAL RESULTS}

Our face recognition system mainly deals with varying illumination conditions, SSS and facial expressions problems. The experimental results have conducted on the "Extended Yale-B face database" which contains illumination variations challenges, JAFFE database which contains facial expressions challenges and AR database which contains facial expressions and difficult illumination conditions, all these databases are standard databases.

\subsection{The experimental Results on Extended Yale-B Database (Varying Illumination Conditions)}

The Extended Yale-B face database is a standard database which provides a perfect test case to testing the systems under varying Illumination conditions, The database contains visual images without pose variations and facial expressions, the total of subjects or persons are 38 subjects, each subject having 64 images photographed under varying illumination conditions [7]. The images in the database are split into five groups according to the direction of the illumination source from the camera axis as described in table 1.
Table 1. Five directions in the Extended YALE B database

\begin{tabular}{|c|c|c|c|}
\hline Subset & Angels & Image numbers & Sample of subset \\
\hline 1 & $\Theta<12$ & 263 & \\
\hline 2 & $20<\Theta<25$ & 456 & \\
\hline 3 & $35<\Theta<50$ & 455 & \\
\hline 4 & $60<\Theta<77$ & 526 & \\
\hline 5 & $85<\Theta<130$ & 714 & \\
\hline
\end{tabular}

The proposed system was tested with only 1 training image per person from the first subset and all the remaining images (2166) for testing. Although there are techniques work well in the case of the availability of training images, these techniques fail if one training sample is available (one image per person) and still needs to be improved to reach the high recognition rate (RR) in case of small sample size. Table 2 shows the results of our system compared with other existing methods in case SSS, these methods were applied to the same database with same feature extraction (RLDA).

Table 2. The experimental results of proposed system compared with other preprocessing methods on extended Yale-B database in case ( SSS).

\begin{tabular}{|c|c|c|c|}
\hline $\begin{array}{c}\text { Training } \\
\text { Image }\end{array}$ & Preprocessing methods & Classifier & $\mathbf{R R}$ \\
\hline \multirow{12}{*}{1} & \multirow{2}{*}{$\begin{array}{l}\text { SSR(Single Scale } \\
\text { Retinex)[13] }\end{array}$} & Cosine & 89.47 \\
\hline & & Correlation & 89.33 \\
\hline & \multirow{2}{*}{$\begin{array}{c}\text { TT(Tan and } \\
\text { Triggs)[2] }\end{array}$} & Cosine & 90.25 \\
\hline & & Correlation & 90.21 \\
\hline & \multirow{2}{*}{$\begin{array}{l}\text { HE(Histogram } \\
\text { equalization)[14] }\end{array}$} & Cosine & 48.75 \\
\hline & & Correlation & 48.75 \\
\hline & \multirow{2}{*}{$\begin{array}{c}\text { CE(Contrast } \\
\text { Equalization)[2] }\end{array}$} & Cosine & 57.11 \\
\hline & & Correlation & 56.28 \\
\hline & \multirow{2}{*}{$\begin{array}{c}\text { DoG+FIT } \\
\text { distribution[15] }\end{array}$} & Cosine & 95.13 \\
\hline & & Correlation & 95.11 \\
\hline & \multirow{2}{*}{$\begin{array}{c}\text { DOG+ Wavelet } \\
\text { with } \mathrm{HE}\end{array}$} & Cosine & $\underline{96.72}$ \\
\hline & & Correlation & $\underline{95.98}$ \\
\hline
\end{tabular}

For further experiments, we have split the database into five groups according to the angle between the illumination source direction and the central camera axis $\left(12^{\circ}, 25^{\circ}, 50^{\circ}, 77^{\circ}, 90^{\circ}\right)$ see table 1, the Experimental results of the proposed system compared with different preprocessing methods on subsets of extended Yale-B database presented in table 3. 
Table 3. The experimental results of proposed system compared with different preprocessing methods on subsets of extended Yale-B database in case (SSS)

\begin{tabular}{|c|c|c|c|c|c|c|}
\hline Methods & Classifiers & 2 & 3 & 4 & 5 & AVG \\
\hline \multirow[b]{2}{*}{ SSR } & cosine & 90.57 & 94.28 & 84.41 & 90.47 & 89.93 \\
\hline & correlation & 89.03 & 94.28 & 82.88 & 88.23 & 88.61 \\
\hline \multirow[b]{2}{*}{ TT } & cosine & 88.38 & 93.85 & 84.22 & 94.39 & 90.21 \\
\hline & correlation & 88.15 & 93.62 & 84.98 & 93.97 & 90.18 \\
\hline \multirow[b]{2}{*}{$\mathrm{HE}$} & cosine & 82.89 & 69.67 & 21.67 & 34.17 & 52.10 \\
\hline & correlation & 82.89 & 69.01 & 19.77 & 35.85 & 51.88 \\
\hline \multirow[b]{2}{*}{$\mathrm{CE}$} & cosine & 85.74 & 74.94 & 22.62 & 52.66 & 58.99 \\
\hline & correlation & 84.64 & 73.84 & 21.67 & 52.10 & 58.10 \\
\hline \multirow{2}{*}{$\begin{array}{c}\text { DoG+ } \\
\text { FIT }\end{array}$} & cosine & 96.17 & 98.11 & 93.90 & 97.00 & 96.30 \\
\hline & correlation & 96.15 & 98.05 & 93.85 & 96.32 & 96.10 \\
\hline \multirow{2}{*}{$\begin{array}{c}\text { DOG+ } \\
\text { Wavelet } \\
\text { with HE }\end{array}$} & cosine & 97.14 & 99.12 & 94.29 & 97.19 & $\underline{96.94}$ \\
\hline & correlation & 95.61 & 98.46 & 94.30 & 96.36 & $\underline{96.18}$ \\
\hline
\end{tabular}

In order to prove the efficiency of the proposed system, 6 images per person were taken as a training set from subset 1 while the remaining subsets are testing sets.Table4 shows the results of the proposed system compared with another existing method [4].

Table 4. Experimental results of proposed system compared with other existing methods (6 training image per person)

\begin{tabular}{|c|c|c|c|c|c|}
\cline { 2 - 6 } \multicolumn{1}{c|}{} & \multicolumn{5}{c|}{ Recognition rates } \\
\hline $\begin{array}{c}\text { Pre-processing } \\
\text { /Features Extraction. }\end{array}$ & $\mathbf{2}$ & $\mathbf{3}$ & $\mathbf{4}$ & $\mathbf{5}$ & AVG \\
\hline $\begin{array}{c}\text { gamma correction. (G) } \\
\text { and brightness } \\
\text { logarithm(Log)+2DDCT }\end{array}$ & 100 & 100 & 100 & 96.0 & 99.0 \\
\hline Misclassified images & 0 & 0 & 0 & 29 & \\
\hline $\begin{array}{c}\text { DoG with wavelet } \\
\text { normalization +HE/ } \\
\text { RLDA - cosine } \\
\text { classifier }\end{array}$ & 100 & 99.8 & 99.6 & 99.3 & 99.7 \\
\hline Misclassified images & 0 & 1 & 2 & 5 & \\
\hline $\begin{array}{c}\text { DoG with wavelet } \\
\text { normalization+HE / } \\
\text { RLDA - correlation } \\
\text { classifier }\end{array}$ & 100 & 99.8 & 99.6 & 99.4 & 99.7 \\
\hline Misclassified images & 0 & 1 & 2 & 4 & \\
\hline No. of test images & 456 & 455 & 526 & 714 & \\
\hline
\end{tabular}

In Table5, the proposed system has compared with other existing methods which provided to solve varying illumination problem. All these methods were applied to the same database "Extended Yale B database" with different feature extractions. In this case, 2166 image was tested with 7 training images. Table 5 shows a comparison of different illumination pre-processing methods with different feature extractions. [3].
Table 5. Comparison of different illumination preprocessing methods on extended YALE-B database (7 training image per person)

\begin{tabular}{|c|c|c|c|c|c|}
\hline \multirow{2}{*}{\multicolumn{2}{|c|}{$\begin{array}{c}\text { Feature } \\
\text { Extraction }\end{array}$}} & \multicolumn{4}{|c|}{ Preprocessing methods } \\
\hline & & HE & SSR & TT & $\begin{array}{c}\text { DoG + } \\
\text { Wavelet } \\
\text { with HE }\end{array}$ \\
\hline \multicolumn{2}{|c|}{ Correlation } & 45.2 & 52.8 & 87.4 & - \\
\hline \multicolumn{2}{|c|}{ Eigen face } & 31.4 & 51.6 & 50.2 & - \\
\hline \multicolumn{2}{|c|}{ LBP } & 62.2 & 58.7 & 90.3 & - \\
\hline \multicolumn{2}{|c|}{ LGBP } & 95.9 & 98.4 & 98.9 & - \\
\hline \multicolumn{2}{|c|}{ Fisherfaces } & 54.8 & 55.5 & 71.6 & - \\
\hline \multicolumn{2}{|c|}{ LEC } & 76.0 & 97.1 & 98.4 & - \\
\hline \multirow{2}{*}{ RLDA } & cosine & 55.5 & 97.0 & 96.2 & 99.45 \\
\hline & correlation & 72.85 & 95.5 & 97.2 & $\underline{99.39}$ \\
\hline
\end{tabular}

\subsection{Experimental Results on JAFFE Database (Facial Expressions Conditions)}

In this Experiments, the JAFFE ("Japanese female facial expressions") database has used for facial expression recognition tasks (FER) [8]. "The database contains ten women images with seven different facial expressions, such as neutral, happy, angry, disgust, fear, sad and surprise as shown in Figure 5. Most of the samples contain three images as examples except some samples. Totally, there are 213 grayscale facial expression images in this database. The performance of our system for each person in the JAFFE database was evaluated by splitting the whole 215 facial images into ten groups according to the persons, each group including all the seven expressions of one distinct person. One image for each expression has selected as training set and the remaining images for testing. Table 6 shows the results of face recognition rates under different facial expressions for each person in case SSS.

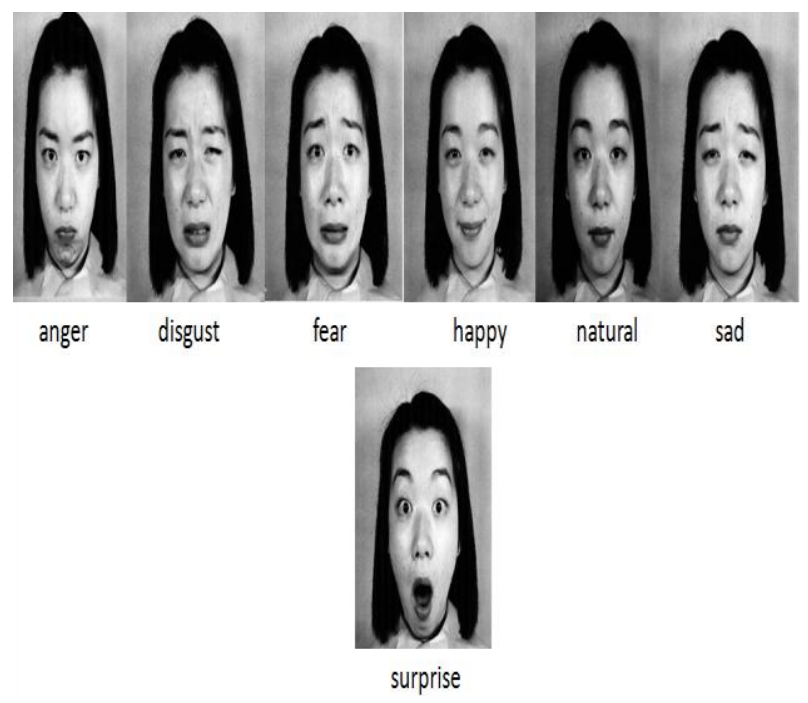

Fig 5 . Sample from JAFFE database 
Table 6. The face recognition rates under different facial expressions for each person on the JAFFE database with one training image

\begin{tabular}{|c|c|c|c|c|}
\cline { 2 - 5 } \multicolumn{1}{c|}{} & \multicolumn{2}{c|}{ RLDA } & \multicolumn{2}{c|}{ PCA-LDA } \\
\hline SUBSETS & cosine & correlation & cosine & Correlation \\
\hline S1 & 86.70 & 73.33 & 86.66 & 73.33 \\
\hline S2 & 86.70 & 80.00 & 73.33 & 73.33 \\
\hline S3 & 86.70 & 100.00 & 93.33 & 86.66 \\
\hline S4 & 76.90 & 76.92 & 69.23 & 69.23 \\
\hline S5 & 78.60 & 71.43 & 92.85 & 92.85 \\
\hline S6 & 78.60 & 71.43 & 64.28 & 50.00 \\
\hline S7 & 84.60 & 84.62 & 76.92 & 76.92 \\
\hline S8 & 85.71 & 78.57 & 85.71 & 85.71 \\
\hline S9 & 78.60 & 71.43 & 71.42 & 78.57 \\
\hline S10 & 86.67 & 80.00 & 73.33 & 73.33 \\
\hline AVG & $\underline{82.98}$ & $\underline{78.77}$ & 78.71 & 75.99 \\
\hline
\end{tabular}

As we note from this experiment that the RLDA achieved appropriate facial expressions recognition rate in the case of small sample size. However, the main challenges for an FER attain the higher classification rates for recognition the expressions. The recognition rates for each individual expression is shown in Table 7 compared with another existing method (PCA with LDA) which provided to solve SSS[16].

Table 7. The recognition rates for each individual expression comparing with another method (PCA-LDA)

\begin{tabular}{|c|c|c|c|c|}
\hline \multirow{2}{*}{$\begin{array}{c}\text { Basic } \\
\text { Expressions }\end{array}$} & \multicolumn{2}{|c|}{ PCA-LDA } & \multicolumn{2}{c|}{ RLDA } \\
\cline { 2 - 5 } & cosine & correlation & cosine & correlation \\
\hline Anger & 85.00 & 95.00 & 100.00 & 100.00 \\
\hline Disgust & 85.00 & 85.00 & 85.00 & 90.00 \\
\hline Fear & 90.90 & 90.90 & 90.90 & 90.90 \\
\hline Happy & 89.47 & 89.47 & 89.47 & 94.73 \\
\hline Natural & 100.00 & 100.00 & 100.00 & 100.00 \\
\hline Sad & 100.00 & 100.00 & 100.00 & 100.00 \\
\hline Surprise & 100.00 & 100.00 & 100.00 & 100.00 \\
\hline AVG & 92.91 & 94.33 & $\underline{95.05}$ & $\underline{96.51}$ \\
\hline
\end{tabular}

In table 8 , we tested our system under different facial expressions by using two images as training set and the remaining images for testing, as mentioned above the database has been divided into ten groups according to the persons, each group including all the seven expressions of one distinct person.

Table 8 shows the face recognition rates of proposed system under different facial expressions for each person with tow training image compared with another existing method (PCA with LDA).
Table 8. The face recognition rates under different facial expressions for each person on the JAFFE database with tow training image.

\begin{tabular}{|c|c|c|c|c|}
\cline { 2 - 5 } \multicolumn{1}{c|}{} & \multicolumn{2}{c|}{ RLDA } & \multicolumn{2}{c|}{ PCA-LDA } \\
\hline SUBSETS & cosine & correlation & cosine & Correlation \\
\hline S1 & 100 & 100.00 & 87.50 & 75.00 \\
\hline S2 & 100 & 100.00 & 87.50 & 87.50 \\
\hline S3 & 100 & 100.00 & 87.50 & 87.50 \\
\hline S4 & 85.71 & 50.00 & 83.33 & 83.33 \\
\hline S5 & 85.71 & 71.43 & 85.71 & 71.42 \\
\hline S6 & 71.42 & 71.43 & 71.42 & 71.42 \\
\hline S7 & 71.42 & 83.33 & 71.42 & 71.42 \\
\hline S8 & 85.71 & 85.71 & 85.71 & 85.71 \\
\hline S9 & 71.42 & 85.71 & 71.42 & 71.42 \\
\hline S10 & 87.50 & 87.50 & 75.00 & 87.50 \\
\hline AVG & $\underline{85.88}$ & $\underline{83.51}$ & 80.65 & 79.22 \\
\hline
\end{tabular}

In order to evaluate the recognition performance of the proposed system under low-resolution cases, the resolution level of the original images $(128 \times 128)$ has been converted to $16 \times 16$. In this experiment, two samples of each expression for each person are used form the training set and the remaining samples are used for testing, the correlation classifier is used for classification. Table 9 shows the results of the proposed system compared with other existing methods [17].

Table 9. The results of proposed system compared with other existing methods on the JAFFE database

\begin{tabular}{|c|c|}
\hline Methods & Recognition Rate (16×16) \\
\hline AMGFR & 56.35 \\
\hline LBP [18] & 68.02 \\
\hline ALBP & 75.39 \\
\hline Tsallis & 63.81 \\
\hline ALBP + Tsallis & 80.40 \\
\hline ALBP + Tsallis + NLDAI & 84.62 \\
\hline RLDA & $\underline{85.86}$ \\
\hline
\end{tabular}

\subsection{Experimental Results on AR Database \\ (Facial Expressions \& Varying Illumination Conditions)}

The AR Face Database [8] contains126 subject (70 Man and 56 women) captured at two different times that mean two sessions. Each session contains 13 images of each subject photographed under varying Illumination conditions with different variations in facial expressions and real disguise. the samples that have changes in illumination with facial expressions were selected only in this experiment (14 images for each person), the total of images are 1400 (50 man and 50 women). Figure 6 shows the samples of this database. The purpose of this experiment testing our system under varying illumination conditions with different facial expressions by using one image as a training sample for all persons in AR database [9], 1300 testing image in total.This is a major challenge to any facial recognition system. Because there are two challenges together, varying illumination conditions with 
different facial expressions. Table 10 shows the recognition rates for all database compared with other existing preprocessing methods in case SSS. These methods were applied to the same database with same feature extraction (RLDA).

Table 10.The recognition rates (Facial Expressions \& Varying Illumination Conditions) on AR database in case (SSS)

\begin{tabular}{|c|c|c|c|c|}
\hline $\begin{array}{c}\text { Training } \\
\text { image }\end{array}$ & methods & classifiers & $\mathbf{R R}$ & $\begin{array}{c}\text { Misclassified } \\
\text { images }\end{array}$ \\
\hline \multirow{12}{*}{1} & \multirow[t]{2}{*}{ SSR } & cosine & 70.46 & 384 \\
\hline & & correlation & 70.07 & 389 \\
\hline & \multirow[t]{2}{*}{ TT } & cosine & 55.30 & 581 \\
\hline & & correlation & 55.23 & 582 \\
\hline & \multirow[t]{2}{*}{$\mathrm{HE}$} & cosine & 70.38 & 385 \\
\hline & & correlation & 70.15 & 388 \\
\hline & \multirow[t]{2}{*}{ CE } & cosine & 63.50 & 550 \\
\hline & & correlation & 60.50 & 500 \\
\hline & \multirow[t]{2}{*}{ DoG+FIT } & cosine & 70.38 & 385 \\
\hline & & correlation & 70.15 & 388 \\
\hline & \multirow{2}{*}{$\begin{array}{c}\text { DOG+ } \\
\text { Wavelet } \\
\text { with HE }\end{array}$} & cosine & $\underline{77.84}$ & 288 \\
\hline & & correlation & $\underline{76.69}$ & 303 \\
\hline
\end{tabular}

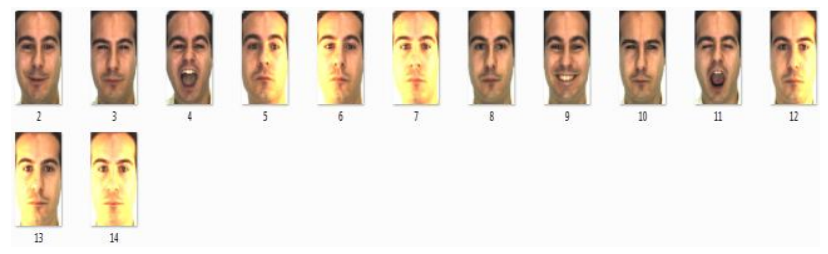

Fig6 .The sample of testing images from AR database

In the last experiment, our system has been tested under varying illumination conditions with different facial expressions by using more than one sample as a training set (7 images per person), 700 testing image in total. Table 11 shows the recognition rates for all databases compared with other preprocessing methods.

Table 11 .The recognition rates (Facial Expressions \& Varying Illumination Conditions) on AR database with 7 images as training set

\begin{tabular}{|c|c|c|c|c|}
\hline $\begin{array}{c}\text { Training } \\
\text { image }\end{array}$ & Methods & classifiers & $\mathbf{R R}$ & $\begin{array}{c}\text { Misclassified } \\
\text { images }\end{array}$ \\
\hline \multirow{12}{*}{7} & \multirow[t]{2}{*}{ SSR } & cosine & 79.85 & 141 \\
\hline & & correlation & 79.71 & 142 \\
\hline & \multirow[t]{2}{*}{$\mathrm{TT}$} & cosine & 62.00 & 266 \\
\hline & & correlation & 61.57 & 269 \\
\hline & \multirow[t]{2}{*}{$\mathrm{HE}$} & cosine & 88.85 & 78 \\
\hline & & correlation & 88.71 & 79 \\
\hline & \multirow[t]{2}{*}{$\mathrm{CE}$} & cosine & 82.28 & 124 \\
\hline & & correlation & 82.14 & 125 \\
\hline & \multirow[t]{2}{*}{ DoG+FIT } & cosine & 87.14 & 90 \\
\hline & & correlation & 87.00 & 91 \\
\hline & \multirow{2}{*}{$\begin{array}{c}\text { DoG+ } \\
\text { Wavelet } \\
+\mathrm{HE}\end{array}$} & cosine & 91.00 & 63 \\
\hline & & correlation & 90.28 & 68 \\
\hline
\end{tabular}

\section{COMPUTATION TIME}

Computation time is a critical factor in many face recognition systems. The proposed system consists of fast and efficient processing chain .Also, RLDA which is considered as effective and fast feature extraction especially in the case of small sample size In addition fast classifiers (cosine, correlation).Table12 shows the Computation time of the proposed system on different databases.

Table12. Computation time of the proposed system on different databases

\begin{tabular}{|c|c|c|c|}
\cline { 2 - 4 } \multicolumn{1}{c|}{} & \multicolumn{3}{c|}{ DATABASES } \\
\hline Time & $\begin{array}{c}\text { Extended Yale } \\
\text { B }\end{array}$ & JAFFE & AR \\
\hline $\begin{array}{c}\text { Training } \\
\text { time }\end{array}$ & $\begin{array}{c}7 \text { sec (1 training } \\
\text { image }-38 \\
\text { images in total) }\end{array}$ & $\begin{array}{c}2 \sec (1 \text { training } \\
\text { image } 10 \\
\text { image })\end{array}$ & $\begin{array}{c}8 \mathrm{sec}(100 \\
\text { image })\end{array}$ \\
\hline $\begin{array}{c}\text { Testing } \\
\text { time }\end{array}$ & $\begin{array}{c}60 \mathrm{sec}(\text { all } \\
\text { database }) \\
2166 \text { images }\end{array}$ & $\begin{array}{c}20 \mathrm{sec} 203 \\
\text { images }\end{array}$ & $\begin{array}{c}70 \mathrm{sec}(1400 \\
\text { images })\end{array}$ \\
\hline
\end{tabular}

Table 13 Default parameters for preprocessing chain

\begin{tabular}{|c|c|c|c|}
\hline pp & Procedure & Parameters & Values \\
\hline DoG+ & DoG & $(\mathrm{img}, \mathrm{s} 1, \mathrm{~s} 2)$ & (img,1, 2) \\
\hline $\begin{array}{c}\text { wavelet + } \\
\text { HE }\end{array}$ & DWT & $\begin{array}{c}\text { (img,s1,haar,post } \\
\text {-preprocessing) }\end{array}$ & (img, 1 ,haar,0) \\
\hline
\end{tabular}

\section{Where:}

- Img: input image.

- $\mathbf{S 1}=[0,1]$ pixels, low frequency filtering parameter and $\mathbf{S 2}$ $>\mathbf{1}$ pixels, high frequency filtering parameter.

- DWT: the procedure which decomposes the image to subbands, then we used HE to processed the LL sub-band.

The experiments were applied using laptop core 7 by MATLAB 2015.we have provided the user by an interface illustrated in figure 7 ,by using this interface, the user can calculate and display the training and testing time with some curves to show the recognition rates.

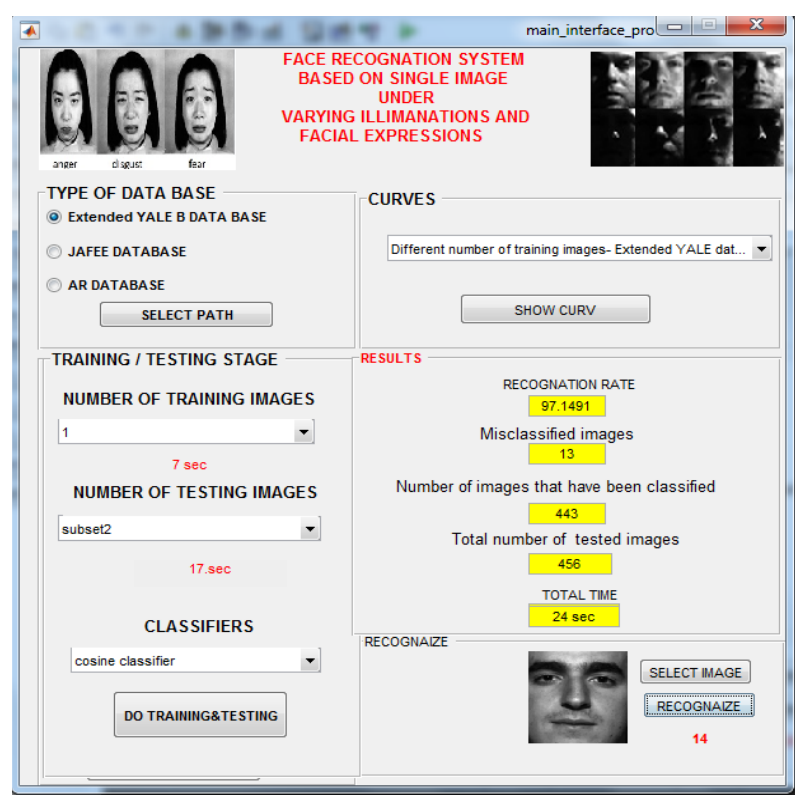

Fig7 The Main İnterface of Proposed System

\section{CONCLUSION}

Many existing methods in a face recognition field still facing challenges such as illumination changes, small sample size 
(SSS) and facial expression, etc., where the main goal of any face recognition system is to develop a system has high recognition rate with short executing time, this can be achieved only through development of a system to solve these problems that mentioned above.

In this paper, we have proposed a system based on the single image as a training set under varying illumination conditions and facial expressions. The simple and fast preprocessing chain have applied with robust feature extraction (RLDA), where RLDA very effective when a small number of training samples is available. The extracted features have classified by using different distance classifiers (cosine, correlation classifiers)

The preprocessing chain consists of DoG with wavelet-based normalization and histogram equalization technique, where DoG filtering firstly applied to reduce the local variations in visible face image and effects of noise, in addition to enhance the edges, then Wavelet based normalization technique is used to obtain an illumination invariant representation of the facial image, where this technique split the image to four sub-bands (LL,LH,HL,LL), the last step of chain is normalize the LL sub-bands by histogram equalization technique to enhance the quality of image and distribute the density of pixels in the image appropriately .

Our proposed system addressed three important problems in the face recognition area at the same time, a small training sample size, varying illumination conditions, and facial expressions, the results mentioned above showed efficiency and effectiveness of the proposed system. Also, many methods disregarded the time factor which is an important factor in real-time systems, our proposed system has a high performance in terms of accuracy of the results, ease of use and low processing time, which saves time and effort. Our experiments were performed by using three standard databases ("Extended Yale- B database", JAFFE database, AR database).

\section{FUTURE WORK}

Since face recognition systems that use visual images are sensitive to illumination changes, facial expressions, occlusions, etc. Many solutions have been suggested to overcome these disadvantages. One of these solutions is Infrared (IR) which not affected by illumination changes and facial expressions because the Infrared (IR) depends on the distribution of blood vessels under the skin. On the other hand, IR still has a problem with temperature changes. In our future work, we will fuse IR with visual images to enhance the performance of face recognition systems by using fast and efficient techniques in wavelet transform domain.

\section{REFERENCES}

[1] Tan, X., Chen, S., Zhou, Z., andZhang., F.2006. Face Recognition from a Single Image per Person: A Survey, $1-34$.

[2] Tan, X., andTriggs, B. (2007). Enhanced Local Texture Feature Sets for Face Recognition Under Difficult Lighting Conditions, 168-182.
[3] Han, H., Shan, S., Chen, X., and Gao, W. (2013). A comparative study on illumination preprocessing in face recognition. Pattern Recognition, 46(6), 1691-1699.

[4] Forczmański, P., Kukharev,G., and Shchegoleva,L. 2012. "An Algorithm of Face Recognition under Difficult Lighting Conditions." Electrical Review (10):201-4.Retrieved (http://red.pe.org.pl/articles/2012/10b/53.pdf).

[5] Shan, C., Gong, S., and Mcowan, P. W. 2009. Facial expression recognition based on Local Binary Patterns : A comprehensive study. Image and Vision Computing

[6] Kumari, J., Rajesh, R., and Pooja, K. M. 2015. Facial expression recognition: A survey. Procedia - Procedia Computer Science,58, 486-491. https://doi.org/10.1016

[7] Lee, K., Ho, J., and Kriegman, D. 2005.Acquiring Linear Subspaces for Face Recognition under Variable Lighting, $1-34$.

[8] Lyons, M. J. 1998. Coding Facial Expressions with Gabor Wavelets, (May), 2-8.

[9] Benavente, R.,and Barcelona, U. A. De. 1999. The AR Face Database Abstract.

[10] Marr, D., Hildreth, E., Series, L., and Sciences, B. 1980 Theory of edge detection, 207(1167), 187-217.

[11] Wang, S. 2012 .An Improved Difference of Gaussian Filter in Face Recognition, 7(6), 429-433.

[12] Iit, E. C. E. n.d. "Multi-Resolution Analysis MultiResolution Analysis : Discrete Wavelet Transforms."

[13] Lu, Juwei, K. N. Plataniotis, and A. N. Venetsanopoulos. 2005. "Regularization Studies of Linear Discriminant Analysis in Small Sample Size Scenarios with Application to Face Recognition." 26:181-91.

[14] Bogdanova,v.. (2010). Image Enhancement Using Retinex Algorithms and Epitomic Representation .10(3) $10-19$.

[15] Krutsch, R., \& Tenorio, D. 2011. Histogram Equalization.

[16] Algharib, A. M. S., Baba, A. 2016. FACE RECOGNITION WITH ILLUMINATION VARYING, 2(1), 150-166.

[17] Sahoolizadeh, A. H., Heidari, B. Z., andDehghani, C. H. 2008. A New Face Recognition Method using PCA, LDA and Neural Network, 2(5), 1354-1359.

[18] Liao, S., Fan, W., Chung, A. C. S., and Yeung, D. 2006. FACIAL EXPRESSION RECOGNITION USING ADVANCED LOCAL BINARY PATTERNS , TSALLIS ENTROPIES AND GLOBAL APPEARANCE FEATURES

[19] Ahonen, T., Hadid, A., and Pietik, M. 2004. Face Recognition with Local Binary Patterns, 469-481. 